\title{
La chirurgie augmentée à Grenoble
}

La réussite d'un partenariat entre scientifiques, cliniciens et industriels au service des patients

Philippe Cinquin et Jocelyne Troccaz

\section{(2) OpenEdition}

\section{Journals}

Édition électronique

URL : https://journals.openedition.org/histoire-cnrs/9098

DOI : 10.4000/histoire-cnrs.9098

ISSN : 1955-2408

Éditeur

CNRS Éditions

\section{Édition imprimée}

Date de publication : 5 octobre 2009

ISSN : 1298-9800

Référence électronique

Philippe Cinquin et Jocelyne Troccaz, "La chirurgie augmentée à Grenoble », La revue pour l'histoire du CNRS [En ligne], 24 | 2009, mis en ligne le 05 octobre 2009, consulté le 20 mai 2021. URL : http://

journals.openedition.org/histoire-cnrs/9098; DOI : https://doi.org/10.4000/histoire-cnrs.9098

Ce document a été généré automatiquement le 20 mai 2021.

Comité pour l'histoire du CNRS 


\title{
La chirurgie augmentée à Grenoble
}

\author{
La réussite d'un partenariat entre scientifiques, cliniciens et industriels
} au service des patients

Philippe Cinquin et Jocelyne Troccaz

1 Au début des années 1980, la robotique a fait son apparition dans les blocs opératoires. L'idée était simple : un robot jusqu'alors à usage industriel ne pouvait-il pas guider la main du chirurgien sur la base de données d'imagerie tridimensionnelle, voire réaliser une partie de l'intervention? À l'origine, il existait la neurochirurgie mini invasive, première utilisatrice métrologique de l'image et des systèmes de repérage mécaniques, les cadres stéréotaxiques. Il s'agissait donc de tirer profit de la précision du robot et de sa capacité à extraire ses informations de positionnement d'une imagerie numérique.

Grenoble fut l'un des tout premiers centres au monde à développer un tel robot et à l'utiliser en routine clinique sur un nombre très conséquent de patients. L'équipe GMCAO, alors dirigée par Philippe Cinquin, fut à l'origine du système développé dans le cadre d'une collaboration avec Alim-Louis Benabid, professeur dans le service de neurochirurgie du CHU de Grenoble. Le système développé par Stéphane Lavallée fut utilisé pour la première fois sur un patient en 1989, puis sur plus d'un millier d'autres patients. Ce système et sa version industrielle (Neuromate, développée dès 1993 par la société Innovative Medical Machines International) permettaient d'opérer en plaçant avec une précision millimétrique dans les structures cérébrales quelquefois profondes, par une voie mini invasive planifiée grâce à des informations issues de l'imagerie (IRM, angiographies cérébrales, etc.), un instrument tel qu'une électrode de stimulation ou de mesure ou un trocart à biopsie. Les pathologies concernées : la maladie de Parkinson, l'épilepsie, les pathologies tumorales... Au-delà de l'approche robotisée du geste médical, des systèmes dits de navigation ont également été conçus à la même époque pour guider le geste principalement dans le domaine de la neurochirurgie puis dans celui de l'orthopédie.

La chirurgie « augmentée »

3 L'objectif poursuivi encore aujourd'hui à Grenoble est de développer des systèmes permettant aux cliniciens d'utiliser une information multi-modale de façon quantitative afin de planifier, de simuler et de réaliser avec précision et en toute 
sécurité des gestes diagnostiques ou thérapeutiques mini invasifs. La problématique scientifique ainsi générée relève de différents domaines tels que le traitement des images médicales, la fusion de données, la modélisation des organes et de leurs comportements ainsi que la simulation et la planification de gestes médicaux, la robotique médicale, l'instrumentation biomédicale, etc. Cette recherche pluridisciplinaire fédère des compétences scientifiques variées avec une dimension applicative extrêmement finalisée.

Ce domaine a évolué au fil des applications et des progrès scientifiques. En effet, si les premiers systèmes se sont souvent focalisés sur des structures anatomiques considérées comme indéformables (en neurochirurgie stéréotaxique et en orthopédie), de nouvelles cibles ont été visées en matière de chirurgie des tissus mous (cardiaque, digestif, vasculaire, urologie...). De nouvelles approches robotiques ont été développées, laissant plus de place à l'interaction avec le clinicien. Ainsi, dans les systèmes de téléopération, l'opérateur déplace un système dit "maître ", et un robot "esclave " reproduit le geste avec une éventuelle fonction de transfert (intégrant un changement d'échelle des forces ou des déplacements et un filtrage des tremblements). Les systèmes dits synergiques ou d'empêchement limitent quant à eux, le geste du clinicien en fonction du planning préétabli.

5 Récemment, les robots multifonctions de type bras anthropomorphiques issus de la robotique « classique » ont également vu l'apparition de systèmes concurrents, dédiés à une application ou à une classe d'applications. Ces nouveaux robots souvent miniaturisés peuvent être fixés directement sur le corps du patient et suivre naturellement ses mouvements éventuels. C'est le cas du robot Viky développé par le laboratoire TIMC et commercialisé par la société Endocontrol Medical. Certains de ces systèmes peuvent même être introduits dans le corps du patient pour y acquérir des données de façon aussi peu invasive que possible ou y délivrer des médicaments. L'aspect dynamique de déformation et de mouvement des tissus mous a également donné lieu à de nombreux travaux en termes d'imagerie, voire de boucles d'asservissement du robot guidé par une imagerie temps réel. Des modèles essaient également de capter la variabilité des organes, de leurs formes et de leurs mouvements. De tels modèles nourrissent d'une information a priori des algorithmes de traitement de l'image ou du signal et peuvent permettre à des systèmes de guidage du geste de se synchroniser avec une cible mobile. Ainsi le système cyberknife de radiothérapie robotisée suit les mouvements d'une tumeur mobilisée par la respiration du patient et garantit ainsi la poursuite de la cible en dépit de ses mouvements. Si ce domaine d'activité était très confidentiel en France il y a vingt ans lors des premiers tests du robot de neurochirurgie à Grenoble, l'application médicale est devenue, au fil des ans, très attractive et de nombreuses équipes françaises de qualité ont peu à peu élargi leur spectre d'activité à ce domaine. En témoignent les nombreux journaux et conférences, les projets soutenus sur ce thème, les différents appels d'offres ainsi que les efforts de structuration de la communauté scientifique dans des groupements de recherche (GDR) par exemple.

De la difficulté à franchir le pas...

6 Cependant, si la recherche en GMCAO est très féconde en France et dans le monde, peu des résultats obtenus dans les laboratoires ou lors d'études précliniques effectuées sur quelques cas sont réellement transférés à la clinique et utilisés sur des populations significatives de patients. Deux difficultés subsistent : 
7 - transformer un produit de laboratoire en un produit industriel utilisable en routine clinique, répondant aux contraintes légales en vigueur et réussissant toutes les étapes de l'évaluation sur fantôme, pièces anatomiques et/ou animaux, patients ;

8 qualité des soins délivrés (i. e. l'amélioration d'un taux de survie), ou en termes de qualité de vie du patient (réduction de la morbidité ou de la mortalité induites, réduction des complications...). Elle peut s'exprimer sur un plan plus économique et organisationnel, par exemple par le raccourcissement des séjours en hôpital et des durées de convalescence. Cette évaluation est un processus complexe nécessitant une traçabilité des soins de leur tout début à leur issue. Dans certaines spécialités, une telle évaluation peut nécessiter le suivi de cohortes de patients pendant plus de 10 ans. Le développement de prototypes préindustriels servant à la validation clinique des concepts est donc primordial. Il est apparu très tôt aux acteurs grenoblois scientifiques et cliniciens qu'un partenariat industriel était essentiel à l'évaluation en "vraie grandeur" des concepts et des outils ainsi que la protection de la propriété intellectuelle de ces approches innovantes. De nombreux brevets ont ainsi été déposés et plusieurs entreprises (six à ce jour) ont été créées pour exploiter ce savoir-faire. Grenoble a également su organiser sa recherche clinique par la création en son sein de structures telles que le Centre d'investigation clinique (avec en particulier sa composante « Innovation technologique »), affilié au CHU et à l'Inserm.

10 C'est bien de l'existence et de la vivacité de ce triptyque industrialo-hospitalouniversitaire très fécond que dépend la réussite de tels projets au-delà de la pertinence de la question médicale posée et de la qualité des réponses techniques qui y sont données.

Système de navigation chirurgicale

Un tel système fonctionne sur un principe proche de celui de nos navigateurs automobiles à base de GPS. Le système de navigation intègre un localisateur optique, magnétique, mécanique - qui permet de suivre en temps réel la position d'un objet (instrument chirurgical, capteur ou même structure anatomique) avec une précision le plus souvent submillimétrique. Sur la base d'un planning réalisé avant l'intervention par exemple sur des données scanner, et après transfert de ce planning à la situation interventionnelle par fusion de données, le système visualise sur un écran la position de l'instrument chirurgical par rapport à une position idéale correspondant au planning et/ou par rapport aux données anatomiques préenregistrées. Le clinicien utilise cette information de localisation pour contrôler son geste et réaliser la stratégie planifiée.

La chirurgie augmentée

L'enjeu n'est plus maintenant de démontrer la faisabilité et l'intérêt de l'introduction d'ordinateurs et de robots au bloc opératoire, mais de mettre au coeur des recherches la qualité du geste interventionnel, quel que soit son support matériel (procédure chirurgicale, radiologique, radiothérapeutique ou endoscopique, vectorisation d'agents thérapeutiques). Pour augmenter cette qualité, et donc le service médical attendu et le service médical rendu, il est indispensable de s'appuyer sur une modélisation de l'expertise et de l'action de l'opérateur médical, ce qui permet de concevoir, de développer et d'évaluer des outils qui augmentent le rapport efficacité/morbidité de son geste. L'augmentation visée est donc d'abord médicale (service médical rendu) et secondairement technique (méthodes et outils d'aide à la planification et à la réalisation de l'intervention). 


\section{RÉSUMÉS}

À l'heure où la technologie est de plus en plus prégnante dans la pratique de soins et où il arrive que des robots assistent le clinicien dans la réalisation de tâches diagnostiques ou thérapeutiques, beaucoup ignorent, qu'ils soient scientifiques, cliniciens ou simples citoyens, que cette histoire a débuté il y a près de 25 ans et que les acteurs français du domaine y ont joué une place de tout premier ordre.

\section{AUTEURS}

\section{PHILIPPE CINQUIN}

Philippe Cinquin, professeur, praticien hospitalier, fondateur et membre de l'équipe GMCAO, est le coordinateur scientifique du Centre d'investigation clinique- innovation technologique du CHU de Grenoble.

\section{JOCELYNE TROCCAZ}

Jocelyne Troccaz, directrice de recherche au CNRS, est responsable de l'équipe Gestes médico-chirurgicaux assistés par ordinateur (GMCAO) au laboratoire « Techniques de l'ingénierie médicale et de la complexité - informatique, mathématiques et applications de Grenoble » (TIMCIMAG). 